\title{
A CHALLENGING CASE OF LIVEDOID VASCULITIS SECONDARY TO SYSTEMIC LUPUS ERYTHEMATOSUS - CASE REPORT
}

\begin{abstract}
Marília Simões Bianchini ${ }^{1, \star}$, Rachel Campos Ornelas ${ }^{1}$, Vinícius Rodrigues Faria ${ }^{1}$, Talita Ramos Siqueira ${ }^{1}$, Adriele Debortoli da Silva ${ }^{1}$, Nícolas Emanuel Oliveira Reis ${ }^{1}$, Pedro Bonifácio Fernandes Junior ${ }^{1}$, Felipe Feliciano Lana ${ }^{1}$, Gabriel Andrade Diniz ${ }^{1}$, Giulia Marilac Teixeira da Silva ${ }^{1}$, João Lucas Guelber Correa ${ }^{1}$, Pollyanna Rafaela de Sena Resende ${ }^{1}$
\end{abstract}

1.Universidade Federal de Juiz de Fora, Juiz de Fora (MG), Brazil.

*Corresponding author: marilia_bianchini27@yahoo.com.br

\section{BACKGROUND}

Livedoid vasculitis (LV) is a chronic pauci-inflammatory or noninflammatory dermatosis that affects blood vessels of dermis, leading to their occlusion, and has a painful manifestation. Its etiology is poorly understood, and it may be associated with prothrombotic events. Lesions, which have maculopapular and erythematous-purpuric characteristics, predominate in the lower limbs, especially in ankles and feet, and ulcerate, leaving an atrophic scar. Several diseases can be associated with LV, such as thrombophilias, neoplasms and autoimmune diseases, such as systemic lupus erythematosus (SLE), rheumatoid arthritis, antiphospholipid antibody syndrome (APS) and systemic sclerosis.

\section{CASE REPORT}

In early January 2018, a 59-year-old white male patient reported the appearance of lesions on the lower third of lower limbs (violaceous, pruritic, painful, nondesquamative macules) that developed into ulcers one week after the onset of symptoms. The lesions were infiltrative, with necrotic background and irregular borders (Fig. 1). The patient had no compatible history with autoimmune disease or neoplasia.

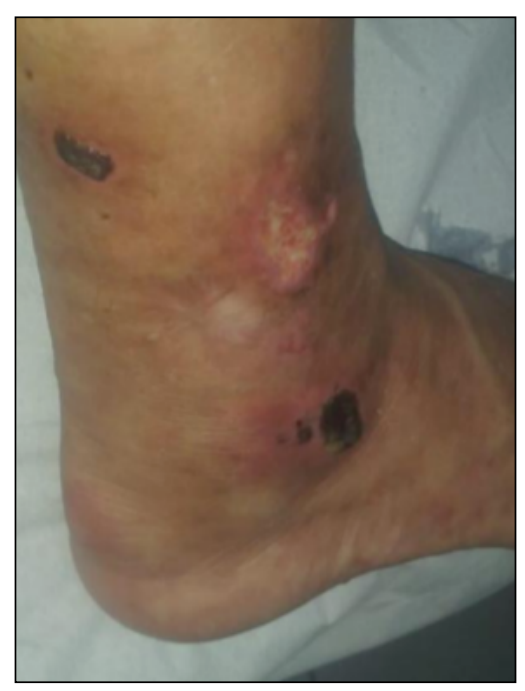

Figure 1. Lesions before treatment.

On consultation, the patient presented unaltered pulmonary, cardiovascular and abdominal physical exams, and normal vital signs. Laboratory tests were performed, ruling out neoplasia, but revealing a reactive ANF 1:160, with fine speckled pattern; anticoagulant in two tests; negative ANCA and low C3 and C4. Infectious serology exams (hepatitis B, C, VDRL, and anti-HIV) were negative. The skin biopsy was compatible with white atrophy or LV. Histological sections showed epidermis with hyperkeratosis, hypergranulosis and spongiosis; and dermis with vasocongestion, edema, mild lymphocytic infiltrate from perivascular and interstitial location, endothelial proliferation, fibrin deposition and intraluminal thrombus formation. 


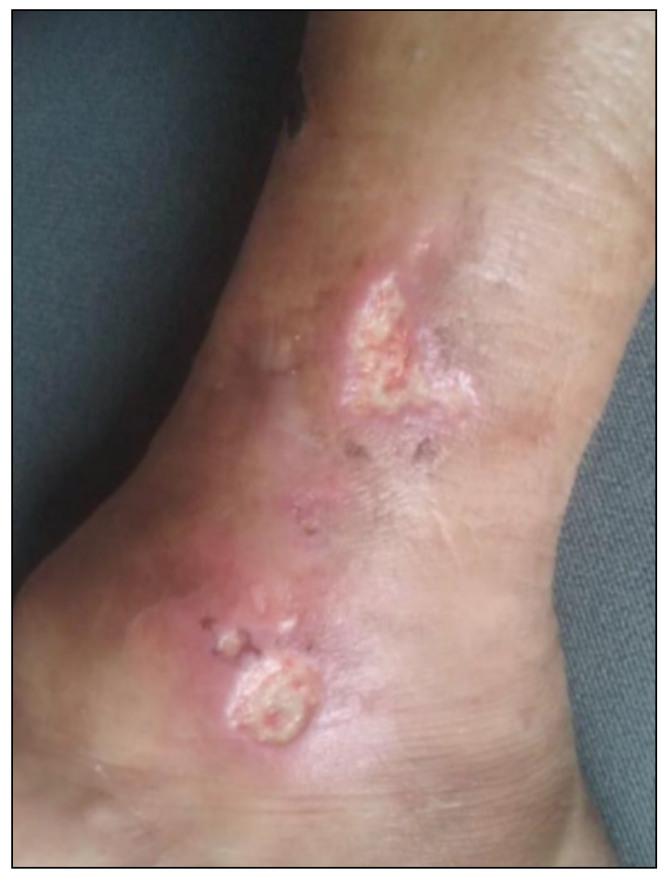

Figure 2. Lesions after treatment with enoxaparin.

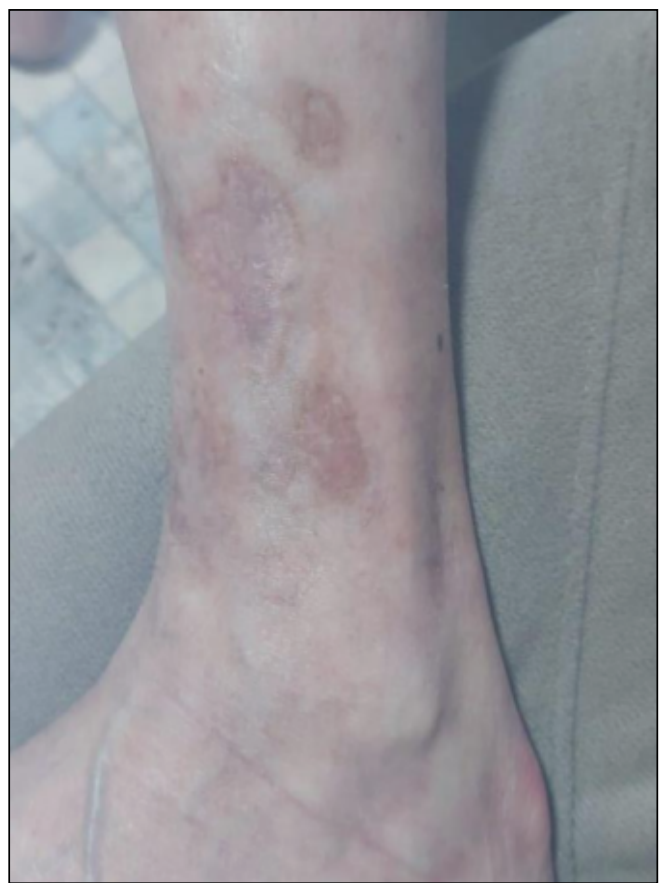

Figure 3. Lesions after treatment with pulse therapy.

\section{CONCLUSION}

Livedoid vasculitis can be classified as primary, when there are no associated diseases, or secondary to other diseases, such as SLE or APS. The diagnosis of LV remains a challenge to rheumatologists, considering that it is based on clinical and histopathological criteria, and the treatment contains anticoagulation, with possibility of including immunosuppressive agents to achieve good clinical response.

\section{KEYWORDS}

Skin diseases, Vascular, Leg ulcer, Immunosuppressive agents. 